\title{
The Synergistic Antitumor Activity of Chidamide in Combination with Bortezomib on Gastric Cancer
}

This article was published in the following Dove Press journal: OncoTargets and Therapy

Purpose: The aim of this study was to investigate the antitumor effect of chidamide in combination with bortezomib on gastric cancer cell lines.

Materials and Methods: First, the sensitivity and $\mathrm{IC}_{50}$ values of chidamide and bortezomib in several gastric cancer cell lines (MGC-803, BGC-823, SGC-7901, and MKN45) were measured using the CCK- 8 assay. Then, the relatively insensitive gastric cancer cell lines (MGC-803 and BGC-823) were treated with low concentrations of chidamide alone, bortezomib alone, or chidamide and bortezomib combination to detect the effects on cell proliferation, apoptosis, migration, and invasion. Finally, the inhibitory effect of the combined chidamide and bortezomib treatment on MGC-803 cells was verified in vivo through tumor formation experiments in nude mice.

Results: Compared with low-dose chidamide or bortezomib alone, the low-dose drug combination significantly inhibited the proliferation, migration, and invasion of MGC-803 and BGC-823 cells and induced apoptosis of the cells. The effects of the low-dose chidamide and bortezomib combination reduced the growth on gastric cancer in vivo were investigated by using a subcutaneous tumor mouse model.

Conclusion: Our results suggest that the combination of chidamide and bortezomib can significantly reduce the proliferation, invasion, and migration of MGC-803 and BGC-823 cells, providing a framework for the clinical evaluation of combined therapies for gastric cancers.

Keywords: chidamide, bortezomib, combination drug therapy, gastric cancer

\section{Introduction}

According to the latest data released by the International Cancer Research Center in 2018, gastrointestinal cancer has the highest incidence and mortality rates among all cancers worldwide. The incidence and mortality rates of gastric cancer are $5.7 \%$ and $8.2 \%{ }^{1}$ These tumors are difficult to find because their early symptoms are not obvious, and most of these tumors would have already progressed to the late stage with lymphatic or distal metastasis by the time they are discovered. ${ }^{2}$ Thus, simple surgical treatment cannot aid in full eradication of these tumors. To date, combinational chemotherapy forms the main part of the treatment strategy for advanced gastric cancer with less than $10 \%$ of 5 -year survival rate. ${ }^{3}$ There is an urgent need to find efficient therapeutic agents to advance the treatment.

Histone deacetylase inhibitors (HDACi) have been considered as a potential anticancer drug nowadays. Histone deacetylase (HDAC) plays a pivotal role in
Correspondence: Kai Sun; Yuqing Chen Department of Hematology, Henan Provincial People's Hospital, People's Hospital of Zhengzhou University,

Zhengzhou, Henan 450003, People's

Republic of China

Tel/Fax +86 37l 65580798

Email sunkai@cellscience.org;

henanblood@sina.com 
regulating the level of cellular DNA acetylation and participate in cell growth, differentiation, and apoptosis. ${ }^{4}$ Elevated expression of HADC was found in patients with cancer compared to healthy controls and associated with decreased survival time. ${ }^{5}$ At present, several HDACiS have been approved by the FDA for the treatment of hematologic malignancies, including vorinostat, romidepsin, panobinostat, and belinostat. ${ }^{6,7}$ Studies, which investigate the application of HDACiS in the solid tumor, have also been conducted. However, a recent multicentre, randomised, and double-blind Phase 3 trial study has shown that off-selectivity histone deacetylase inhibitors have adverse reactions such as nausea, diarrhea, fatigue, anorexia, thrombosis and thrombocytopenia. ${ }^{8}$ Fortunately, the production of a new generation of oral histone deacetylase inhibitor Chidamide has changed this dilemma. Chidamide (CS055) is an orally active benzylamide HDAC inhibitor that selectively inhibits the expression of type I and $\mathrm{IIb}$ HDACs $(1,2,3$, and 10), thereby exerting antitumor effects through the promotion of cell cycle arrest and apoptosis. ${ }^{9}$ In addition, Phase I clinical trials of chidamide for the treatment of advanced lymphoma and solid tumors have shown that it is well tolerated, indicating the drug as a promising candidate for combination with a variety of other antitumor drugs. ${ }^{10}$ Indeed, the view has also been confirmed in non-small-cell lung carcinoma, ${ }^{11}$ pancreatic cancer, ${ }^{12}$ and leukemia cell lines. ${ }^{13}$

The proteasome pathway is involved in the course of cell cycle regulation and apoptosis via maintaining the dynamic equilibrium of several regulatory proteins, such as cyclins, transcription factors, oncogenic proteins and tumor suppressors. ${ }^{14}$ Inhibition of proteasome can result in cellular apoptosis by means of a variety of mechanisms and has shown promising anti-tumor effects in hematologic cancers and solid tumors. ${ }^{15,16}$ Bortezomib, the first-inclass proteasome inhibitor, functions as a highly selective and reversible inhibitor of 26S proteasome activity. ${ }^{17}$ It has been approved by the US FDA for the treatment of human multiple myeloma and mantle cell lymphoma. ${ }^{18}$ There are also some studies investigating its anti-tumor activity in ovarian cancer, gastric cancer. ${ }^{19,20}$ More importantly, bortezomib shows synergistic when in combination with other chemotherapeutic agents. ${ }^{21,22}$ Previous studies have suggested that bortezomib could synergize HDACiinduced apoptosis in esophageal cancer cells, multiple myeloma cells and non-small-cell lung cancer (NSCL) cells. ${ }^{19,22,23}$ However, there is no study investigating the synergistic effect in gastric cancer.
To combat the problem of the adverse reactions in treatment and improve the effectiveness of chemotherapy, in our study, we investigated the antitumor effect and possible mechanism of selective histone deacetylase inhibitor Chidamide combined with proteasome inhibitor bortezomib on MGC-803 and BGC-823 cells in vitro and in vivo, hoping to provide more insights for the future application in clinical work.

\section{Materials and Methods Reagents and Antibodies}

Chidamide was provided by Chipscreen Biosciences (Chipscreen Biosciences Co., Ltd., Shenzhen, China) and dissolved in dimethyl sulfoxide (DMSO) (Sigma-Aldrich, St. Louis, MO, USA) at a $100 \mathrm{mM}$ concentration as a stock solution. Bortezomib was purchased from Xian Janssen (Xian Janssen Pharmaceutical Co., Ltd., Xian, China) and dissolved in DMSO at the concentration of $1 \mathrm{mM}$ as a reserve solution. The two solutions were kept at $-80{ }^{\circ} \mathrm{C}$ and diluted to the working concentration in subsequent experiments.

Primary antibodies against B-cell lymphoma 2 (Bcl-2), matrix metallopeptidase 2 (MMP2), E-cadherin and $\mathrm{N}$-cadherin were obtained from Santa Cruz Biotechnology (Santa Cruz, CA, USA). Antibodies against glyceraldehyde 3-phosphate dehydrogenase (GADPH), cleaved caspase-3, acetyl-H3, acetyl-H14, AKT, p-AKT, PI3K, p-PI3K, cleaved-PARP1, and Bad were purchased from Abcam (Cambridge, MA, USA). Antibodies against cyclin D1 and cyclin E1 were purchased from Cell Signaling Technology (Danvers, MA, USA).

\section{Cell Lines and Cell Culture}

The human gastric cancer cell lines MGC-803, BGC-823, SGC-7901 and MKN45 were purchased from the Shanghai Cell Bank of the Chinese Academy of Sciences (Shanghai, China). These cell lines were inoculated into RMPI 1640 medium containing 10\% fetal bovine serum (FBS; Gibco, USA) and a $1 \%$ penicillin-streptomycin mixture (Solarbio Science \& Technology Co., Ltd., Beijing, China), and then cultured under $5 \% \mathrm{CO}_{2}$ in an incubator at $37^{\circ} \mathrm{C}$. The cells were subcultured every 2-3 days, and the exponentially growing cells were used in the experiments.

\section{Cell Viability Assay}

To evaluate the cytotoxic effects of bortezomib and chidamide alone or combined on the gastric cancer cell lines, 
Cell Counting Kit-8 (CCK-8, DOJINDO, Kumamoto, Japan) analysis was carried out in strict accordance with the manufacturer's protocol. The gastric cancer cells were inoculated into respective 96-well plates at a density of $1-3 \times 10^{4} / \mathrm{mL}$ and then exposed to different concentrations of chidamide $(0-50 \mu \mathrm{M})$ or bortezomib $(0-100 \mathrm{nM})$ for 48 hours, following which the standard CCK-8 assay was performed. The cell viability ratio was calculated with the following formula: Cell viability ratio $(\%)=[\mathrm{OD}$ (drug groups) - OD (Blank)]/[OD (Control) - OD $($ Blank) $] \times 100$. The combination index $(\mathrm{CI})$ value was used to assess the degree of drug interaction, where $\mathrm{CI}<1$, $\mathrm{CI}=1$, and $\mathrm{CI}>1$ indicated synergistic, additive, and antagonistic effects, respectively. The statistical analyses and $\mathrm{IC}_{50}$ value determination were carried out using Statistical Package for the Social Sciences Version 22.0 software (SPSS, Inc., Chicago, IL, USA). Each experiment was carried out with four replicates, and the results were calculated from three independent experiments.

\section{EdU-Labeling Assay}

After 48 hours of the various drug treatments, the MGC-803 and BGC-823 cells were cultured overnight in respective 96-well plates at the density of $1 \times 10^{5}$ cells per well. The treated cells were also labeled with 5-ethynyl-2'deoxyuridine (EdU) using the Cell Light Edu Apollo 567 In Vitro Kit (Ribobio Biotechnology Co., Ltd., Guangzhou, China) according to the manufacturer's protocol. Images were captured using an Axio Imager $\mathrm{M}^{2}$ fluorescence microscope (Zeiss, Germany).

\section{Cell Cycle Analyses}

Cell cycle analyses were performed using the Cell Cycle Phase Determination Kit (Cayman Chemical, USA) according to the manufacturer's instructions. Briefly, after 48 hours of the various drug treatments, cells were digested and suspended at a density of $1 \times 10^{6}$ cells $/ \mathrm{mL}$, and fixed and permeabilized at $-20{ }^{\circ} \mathrm{C}$ for 24 hours. Subsequently, cells were stained with propidium iodide and staining solution, which was followed by FACS analysis. Flow cytometry and data analysis were performed using the BD FACScalibur and Cellquest system (BD Bioscience, CA).

\section{Flow Cytometric Analysis}

MGC-803 and BGC-823 cells that had been exposed to bortezomib or chidamide alone or to both combined for 48 hours were collected and stained using the FITC Annexin V Apoptosis Detection Kit (BD Pharmingen
Biosciences, San Diego, CA, USA) and then analyzed by flow cytometry according to the manufacturer's instructions. In brief, the cells were washed twice with phosphate-buffered saline (PBS) and then $400 \mu \mathrm{L}$ of Annexin $\mathrm{V}$ Binding Buffer was added. Thereafter, $5 \mu \mathrm{L}$ of FITC Annexin $\mathrm{V}$ and $5 \mu \mathrm{L}$ of propidium iodide were added to each sample well and the plates were then incubated in the dark for 10 minutes. The samples were finally analyzed by flow cytometry, where the number of apoptotic cells was counted using Furjo software.

\section{Wound Healing Assay}

MGC-803 and BGC-823 cells were resuspended after 48 hours of drug treatment. After adjustment of the cell concentration to $1 \times 10^{5} / \mathrm{mL}, 2 \mathrm{~mL}$ was added to each well of respective 6-well plates. Once the degree of cell fusion had reached $90 \%$, a wound of approximately $1 \mathrm{~mm}$ width was made in the middle of each well using the tip of a $200-\mu \mathrm{L}$ pipette. After discarding the old medium, the cell layer was washed twice with PBS and then $2 \mathrm{~mL}$ of serum-free medium was added. The cells were observed and images of the scratch width at $0 \mathrm{~h}$ were taken under a microscope. After 24 hours, the scratch area was calculated using Image-Pro Plus 6.0 software.

\section{Transwell Migration Assay}

After treatment for 48 hours with the various drugs, the MGC-803 and BGC-823 cells were digested with trypsin containing ethylenediaminetetraacetic acid (Solarbio Science \& Technology Co., Ltd., Beijing, China) and then resuspended in medium without the FBS and penicillin-streptomycin mixture. Subsequently, $200 \mu \mathrm{L}$ of the cell suspension was added to the upper layer of a Transwell chamber (Corning, New York, NY, USA), whereas $500 \mu \mathrm{L}$ of medium containing $10 \%$ FBS was added to the lower chamber. Incubation was then carried out under $5 \% \mathrm{CO}_{2}$ in an incubator at $37{ }^{\circ} \mathrm{C}$ for 24 hours. Then, the upper chamber medium was discarded, and the cells on the surface of the upper chamber that had not passed through were gently wiped after washing twice with PBS. The cells were fixed with $4 \%$ paraformaldehyde for 20 minutes, stained with $0.1 \%$ crystal violet dye for 10 minutes, airdried at ambient temperature, and finally observed and photographed under an optical microscope (Nikon E100; Nikon Corp., Tokyo, Japan). Five high-power microscope fields per well were randomly selected for counting the number of cells that had passed through the bottom 
membrane of the compartment. The results were an average of three experimental repeats.

\section{Transwell Invasion Assay}

For this assay, $100 \mu \mathrm{L}$ of $20 \mathrm{mg} / \mathrm{mL}$ Matrigel (BD Biosciences, San Jose, CA, USA) was melted on ice and then diluted 1:8 with serum-free medium at $4{ }^{\circ} \mathrm{C}$. Thereafter, $50 \mu \mathrm{L}$ of the diluted Matrigel was added to the upper chamber of a Transwell chamber, which was then placed in a $37{ }^{\circ} \mathrm{C}$ incubator for 2 hours. After the gel had solidified, the invasion assay was carried out using the same protocol as used for the cell migration assay.

\section{Western Blot Analysis}

MGC-803 and BGC-823 cells were grown on 6-well culture plates and treated with the various drugs for 48 hours. Next, the total protein of the cells was extracted with RIPA buffer containing a proteinase inhibitor (Beyotime Biotechnology Co., Ltd., Shanghai, China) and then separated by SDS-PAGE (Beyotime Biotechnology Co., Ltd., Shanghai, China). Thereafter, the protein bands were transferred to a polyvinylidene difluoride membrane (Millipore, Bedford, MA, USA). The membrane was blocked with 5\% skim milk for 1 hour, and then incubated with the primary antibody for 1 hour, followed by the horseradish peroxidase-conjugated secondary antibody (Boster, Wuhan, China) for another hour. Using $\beta$-actin as the internal reference, the protein bands were visualized by enhanced chemiluminescence (ECL; New England Biolabs, Ipswich, MA, USA). The gray values of the target proteins and the internal reference strip were analyzed and quantified with ImageJ software.

\section{HDAC Activity Assay}

HDAC activity assay was performed using the colorimetric HDAC activity assay kit from BioVision (BioVision Research Products, USA) according to the manufacturer's instructions. Briefly, after treatment for 48 hours with the various drugs, cells were lysed by RIPA buffer and a total of $100 \mu \mathrm{g}$ of cell lysate was used for the assays. Each tissue sample had its respective blank, ie, without lysine developer. Absorbance was estimated using a 96-well plate reader at $405 \mathrm{~nm}$.

\section{Xenograft Tumor in Nude Mice}

To produce a murine model of gastric tumor, MGC-803 cells $\left(5 \times 10^{6}\right)$ were suspended in $100 \mu \mathrm{L}$ of PBS and inoculated into the subcutaneous tissue of the right flank of 4-week-old BALB/c nude mice (Zhengzhou, China). Once the xenografts had grown to approximately $100 \mathrm{~mm}^{3}$ in size, the mice were randomly divided into four groups [PBS only, $0.5 \mathrm{mg} \cdot \mathrm{kg}^{-1}$ bortezomib only, $25 \mathrm{mg} \cdot \mathrm{kg}^{-1} \cdot \mathrm{day}^{-1}$ chidamide only, and $0.5 \mathrm{mg} \cdot \mathrm{kg}^{-1}$ bortezomib plus $25 \mathrm{mg} \cdot \mathrm{kg}^{-1} \cdot \mathrm{day}^{-1}$ chidamide $\left.(\mathrm{n}=5)\right]$. Chidamide was provided as a solution $\left(25 \mathrm{mg} \cdot \mathrm{kg}^{-1}\right)$ by gastric gavage every day, whereas bortezomib was injected intraperitoneally at every 3 days. The body weight of the mice and the tumor volume were measured at every 3 days. The tumor volume was estimated with the following formula: $\left(\mathrm{L} \times \mathrm{W}^{2}\right) / 2$, where $\mathrm{L}$ is the largest diameter and $\mathrm{W}$ is the diameter perpendicular to $\mathrm{L}$. The mice were sacrificed at 30 days post treatment. All experimental procedures were approved by the Animal Care Ethics Committee of the Zhengzhou University and Henan Provincial People's Hospital, and were performed in accordance with the American Physiology Society's "Guides for the Care and Use of Laboratory Animals" published by the National Institutes of Health.

\section{HE Staining}

HE staining was conducted according to routine protocols. Briefly, after deparaffinization and rehydration, $5 \mu \mathrm{m}$ longitudinal sections were stained with hematoxylin solution for $5 \mathrm{~min}$ followed by 5 dips in $1 \%$ acid ethanol $(1 \% \mathrm{HCl}$ in $70 \%$ ethanol) and then rinsed in distilled water. Then, the sections were stained with eosin solution for $3 \mathrm{~min}$ and followed by dehydration with graded alcohol and clearing in xylene. The mounted slides were then examined and photographed using an Olympus BX53 microscope (Tokyo, Japan).

\section{Statistical Analyses}

The results were analyzed with SPSS 23.0 software. The data were expressed as the means \pm standard deviation (SD). One-way ANOVA analysis of variance was used to assess differences between the groups, where statistical significance was considered at $P<0.05$.

\section{Results}

\section{Antiproliferative Activity of Chidamide} Alone or in Combination with Bortezomib in Human Gastric Cancer

\section{Cell Lines}

In this study, four types of gastric cancer cell lines MGC-803, BGC-823, SGC-7901 and MKN-45 were used. CCK-8 
assays were first employed to evaluate the antiproliferative effects of chidamide and bortezomib as single agents. Both agents alone showed dose-dependent growth inhibitory effects on all four cell lines, with SGC-7901 being particularly sensitive to bortezomib and more sensitive to chidamide than the other three cell lines (Figure 1A). Table 1 summarizes the $\mathrm{IC}_{50}$ values of chidamide and bortezomib in these four cell lines. Because the SGC-7901 cells were very sensitive to bortezomib, this cell line was eliminated in the following experiments, and the other three cell lines (MGC803 , MKN-45 and BGC-823) were treated with varying concentrations of chidamide either in the absence or presence of low-dose bortezomib (15 nM). Compared with the cells treated with chidamide alone, all cells treated with different doses of chidamide plus bortezomib (15 nM) exhibited decreased viability (Figure 1B-D). The CI values were all less than 1 (Table 1), indicating that there was a synergistic interaction between low-dose chidamide and bortezomib in these three cancer cell lines. Because the synergistic effect was more significant in the MGC-803 and BGC-823 cells, these two cell lines were used in the subsequent experiments to determine the possible mechanisms behind the killing effect of the combination drug therapy.

\section{Chidamide Combined with Bortezomib Inhibited the Proliferation of the MGC-803 and BGC-823 Cell Lines}

To study whether the combination of chidamide and bortezomib can inhibit the proliferation of MGC-803 and BGC-823 cells, the cells were treated with the $\mathrm{IC}_{50}$ dose of chidamide $(120 \mu \mathrm{M}$ for MGC-803, and $80 \mu \mathrm{M}$ for BGC-823), a low dose of chidamide $\left(\mathrm{IC}_{50} / 4,30 \mu \mathrm{M}\right.$ for MGC-803, and $20 \mu \mathrm{M}$ for BGC-823), a low dose of bortezomib $(15 \mathrm{nM})$, and a low dose of chidamide combined with bortezomib. The effects of the different treatments on cell proliferation were then evaluated by detecting the proliferation-associated cyclin proteins, EdU staining and Cell-cycle distribution analysis. As shown in Figure 2A and B, compared with the low-dose chidamide treatment, the low dose of chidamide combined with bortezomib significantly inhibited the expression of proliferation-related proteins cyclin D1 and cyclin E1 in the MGC-803 and BGC-823 cells. Moreover, EdU staining revealed that the low-dose drug combination had significantly reduced the proportions of MGC-803 and BGC-823 cells in the proliferative state (Figure 2C and D). Finally, cell cycle analysis showed that low-dose drug combination

A

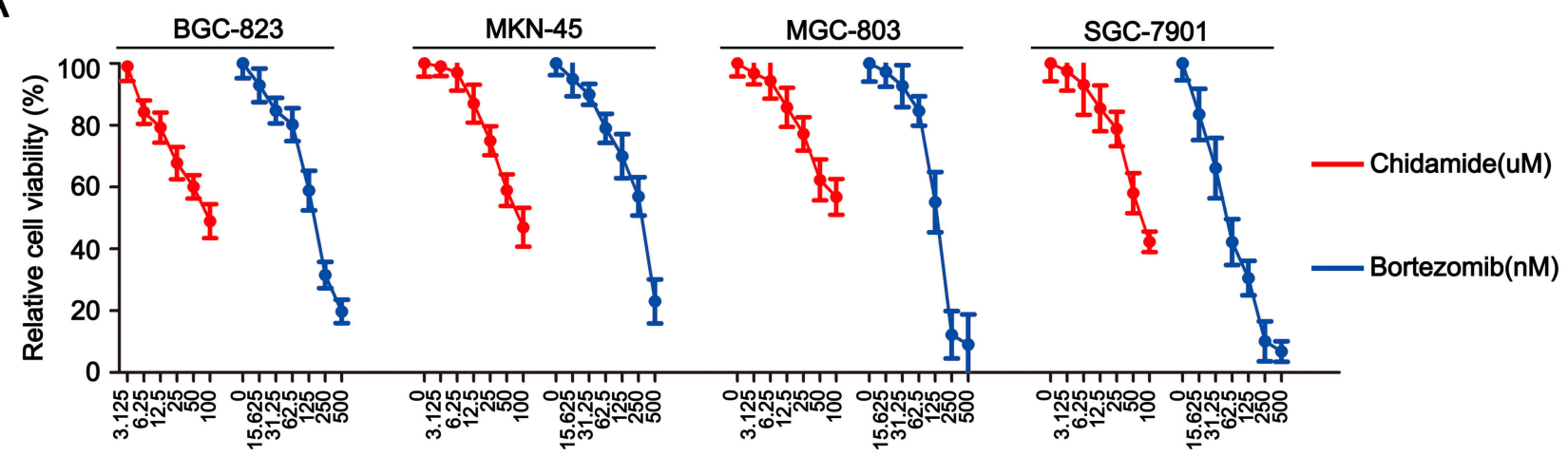

B

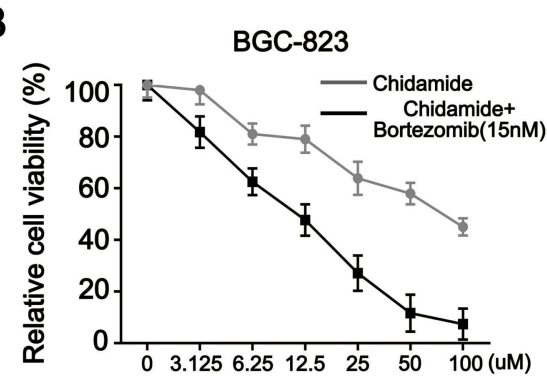

C

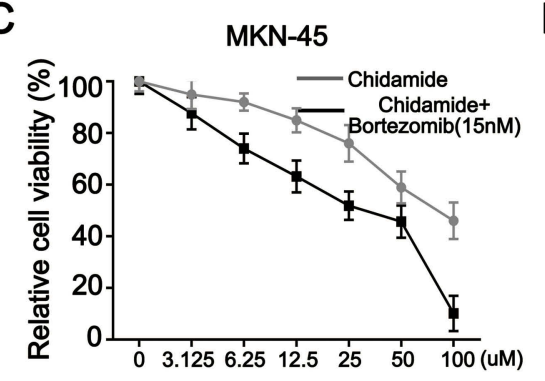

D

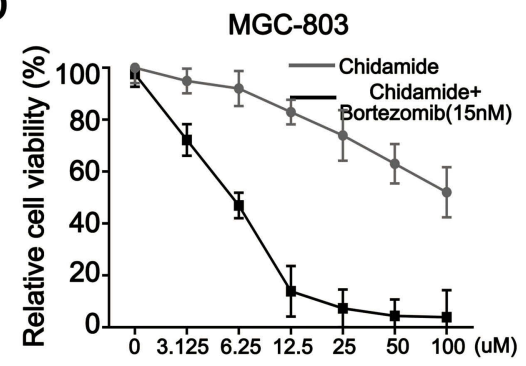

Figure I Antiproliferative activity of chidamide alone or in combination with bortezomib in human gastric cancer cell lines. (A) Representative pairs of cell viability curves for the MGC-803, BGC-823, SGC-790I and MKN45 cell lines after their treatment with serial dilutions of chidamide or bortezomib for 48 hours. (B, C, and D) Representative pairs of cell viability curves for the MGC-803, BGC-823 and MKN45 cell lines after their treatment with serial dilutions of chidamide in combination with bortezomib (I5 nM) for 48 hours. All experiments were repeated at least three times. 
Table I The IC50 Values of Chidamide and Bortezomib in GC and EC Cell Lines

\begin{tabular}{|c|c|c|c|c|}
\hline \multirow[t]{2}{*}{ Cell Lines } & \multirow[t]{2}{*}{ Chidamide IC50 \pm SD $(\mu \mathrm{M})$} & \multicolumn{3}{|c|}{ Bortezomib IC50 \pm SD (nM) } \\
\hline & & No Chidamide & Chidamide & Cl \\
\hline BGC-823 & $80.44 \pm 5.42$ & $|52.67 \pm| 4.67$ & $53.50 \pm 7.33$ & 0.869 \\
\hline MKN-45 & $77.15 \pm 6.76$ & $235.38 \pm 17.49$ & $117.60 \pm 12.55$ & 0.962 \\
\hline MGC-803 & $115.68 \pm 10.583$ & $66.58 \pm 7.33$ & $26.39 \pm 4.29$ & 0.735 \\
\hline SGC-790I & $74.40 \pm 6.14$ & $54.67 \pm 5.49$ & & \\
\hline
\end{tabular}

Abbreviations: $\mathrm{Cl}$, combination index; SD, standard deviation.

could increase the rate of $\mathrm{G}_{0} / \mathrm{G}_{1}$ phase and significantly reduce the rate of $\mathrm{S}$ phase both in the MGC-803 and BGC823 cells (Figure 2E and F). Therefore, we conclude that low-dose drug combination treatment can significantly enhance the inhibitory effect of chidamide on BGC-823 and MGC-803 cell proliferation.

\section{Combination of Chidamide and Bortezomib Synergistically Enhanced Cell Apoptosis in the MGC-803 and BGC-823 Cell Lines}

To investigate the mechanism of cell death induced by chidamide alone or in combination with bortezomib, the changes in apoptosis after drug treatment were examined in MGC-803 and BGC-823 cells. We first examined the effects of chidamide alone, bortezomib alone, or their combination on the expression of apoptosis-related proteins (viz., cleavedPARP, cleaved caspase-3, P53, Bad, and Bcl-2) in the cells. Low-dose chidamide combined with bortezomib significantly induced the expression of the apoptosis-executing protein cleaved-PARP, cleaved caspase- 3 and $\mathrm{Bad}$, and reduced the expression of the apoptosis-inhibiting proteins P53 and Bcl-2 (Figure 3A and B). In addition, we further used flow cytometry to examine the effects of the drugs on MGC-803 and BGC-823 cell apoptosis. As shown in Figure $3 \mathrm{C}$ and $\mathrm{D}$, the low dose of chidamide or bortezomib alone had little effect on the apoptosis of both cell lines, whereas the low dose of chidamide plus bortezomib increased the apoptotic rate of both types of cells significantly. Therefore, we conclude that low-dose drug combination can significantly enhance the effect of chidamide on MGC-803 and BGC-823 cell apoptosis.

\section{Combination of Chidamide and Bortezomib} Synergistically Inhibited the Migratory Abilities of MGC-803 and BGC-823 Cells

The migratory ability is the driving force behind the spread of cancer cells. To assess whether low doses of bortezomib can enhance the ability of chidamide to inhibit cell migration, in vitro Transwell assays and scratch-wound assays were performed. As shown in Figure 4A and B, the Transwell assay showed that low-dose bortezomib had significantly enhanced the inhibitory effect of chidamide on the migratory ability of the MGC-803 and BGC-823 cells. This effect was verified by the scratch-wound assays (Figure $4 \mathrm{C}$ and D). Therefore, we conclude that low-dose bortezomib can significantly enhance the inhibitory effect of chidamide on MGC-803 and BGC-823 tumor cell migration.

\section{Combination of Chidamide and}

\section{Bortezomib Synergistically Inhibited the Invasive Abilities of MGC-803 and BGC-823 Cells}

The invasive ability is an important function for tumor cell infiltration and distant metastasis and colonization, and thus the inhibition of this ability of tumor cells would be of great significance. Therefore, the ability of low-dose bortezomib to enhance the inhibitory effect of chidamide on the invasive abilities of MGC-803 and BGC-823 cells was evaluated. First, through Transwell invasion experiments, it was found that low-dose bortezomib or low-dose chidamide alone could not effectively inhibit the invasive ability of either of the cell lines, whereas low-dose bortezomib combined with low-dose chidamide significantly inhibited invasive ability in both types of cancer cells (Figure 5A and B). We then examined the effects of combined bortezomib and chidamide on the expression of key proteins involved in tumor cell invasion (viz., E-cadherin, N-cadherin, and MMP9). Similar to the results of the Transwell invasion experiment, the low dose of bortezomib significantly enhanced the inhibitory effect of chidamide on the expression of $\mathrm{N}$-cadherin, MMP9, and induced the expression of E-cadherin in the MGC-803 and BGC-823 cells (Figure 5C and D). Therefore, it was concluded that low-dose bortezomib combined with chidamide could significantly inhibit the invasive abilities of the MGC-803 and BGC-823 cell lines. 
A

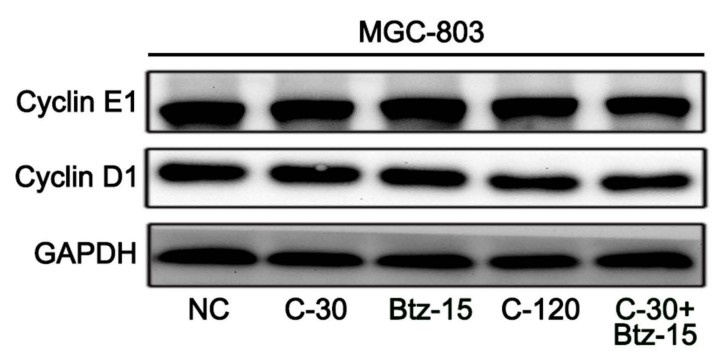

C
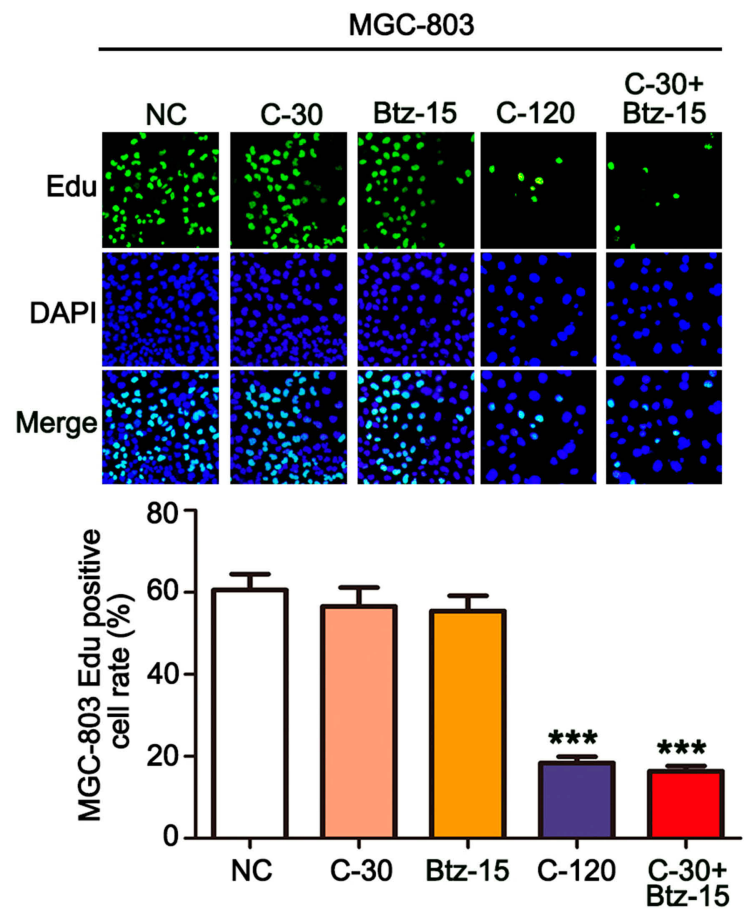

E

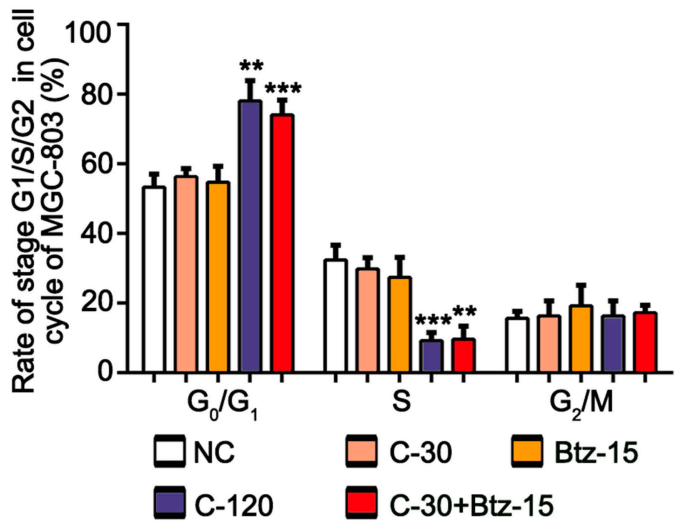

B

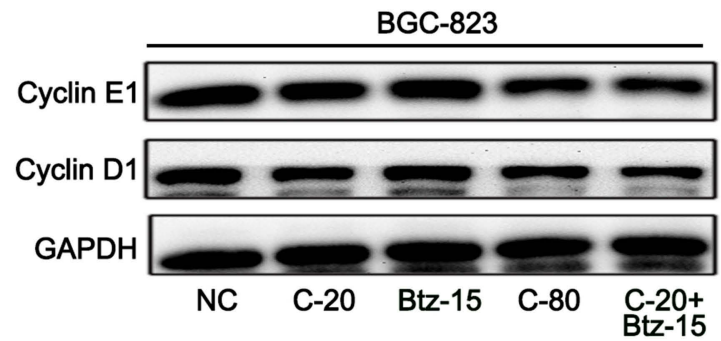

D
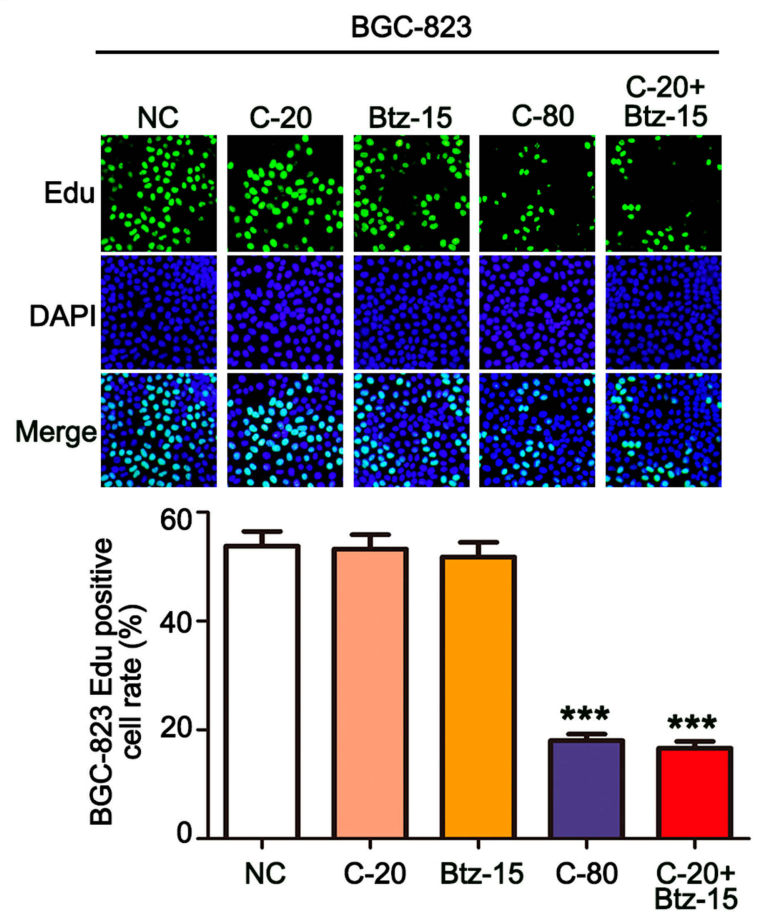

F

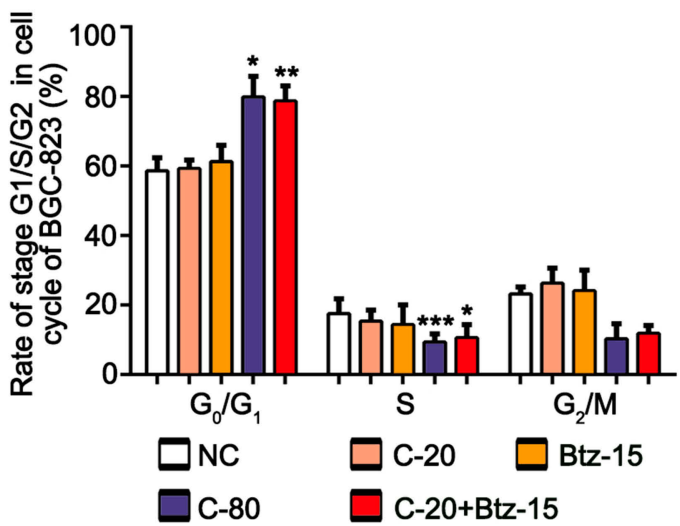

Figure 2 Chidamide combined with bortezomib inhibited the proliferation of the MGC-803 and BGC-823 cell lines. The MGC-803 and BGC-823 cell lines were treated with chidamide (30 $\mathrm{M}$ for MGC-803 or $20 \mu \mathrm{M}$ for BGC-823), bortezomib (I5 nM), chidamide (I $20 \mu \mathrm{M}$ for MGC-803 or $80 \mu \mathrm{M}$ for BGC-823), or chidamide (30 $\mu$ M for MGC-803 or $20 \mu$ M for BGC-823) in combination with bortezomib (I5 nM) for 48 hours. (A and B) Representative images of cyclin DI and cyclin EI expression. (C and D) EdU staining, showing cells in a proliferating state after the different treatments, and their statistical results. (E and F) Cell-cycle distribution of MGC-803 and EC-I09 cells were confirmed after the different treatments by propidium iodide staining and FACS analysis. (One-way ANOVA with Bonferroni's post hoc test was applied to compare the indicated groups. $* P<0.05, * * P<0.01$, and $* * * P<0.001$, compared with the negative control, chidamide-, or bortezomib-alone groups). The experiment was repeated at least three times. Negative control: DMSO. 
A

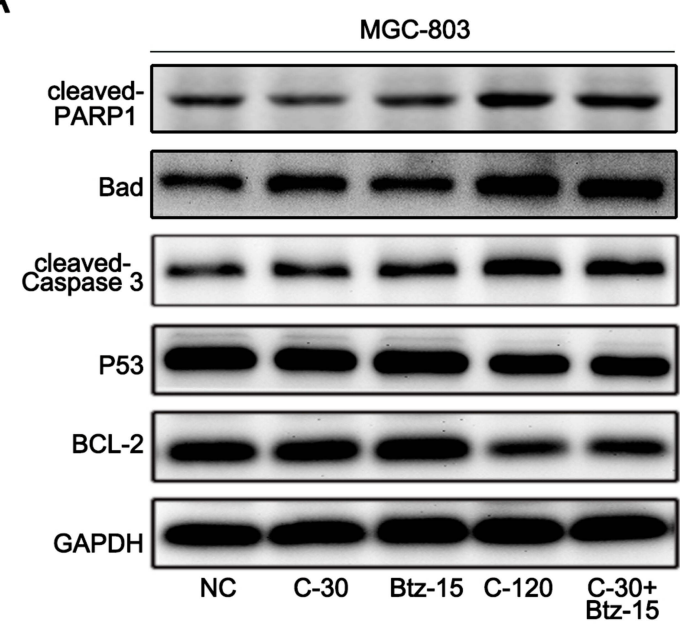

B

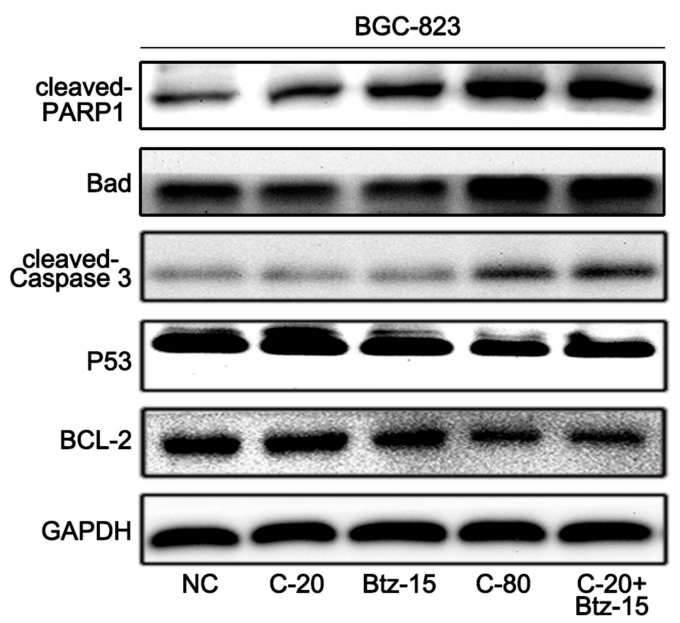

C

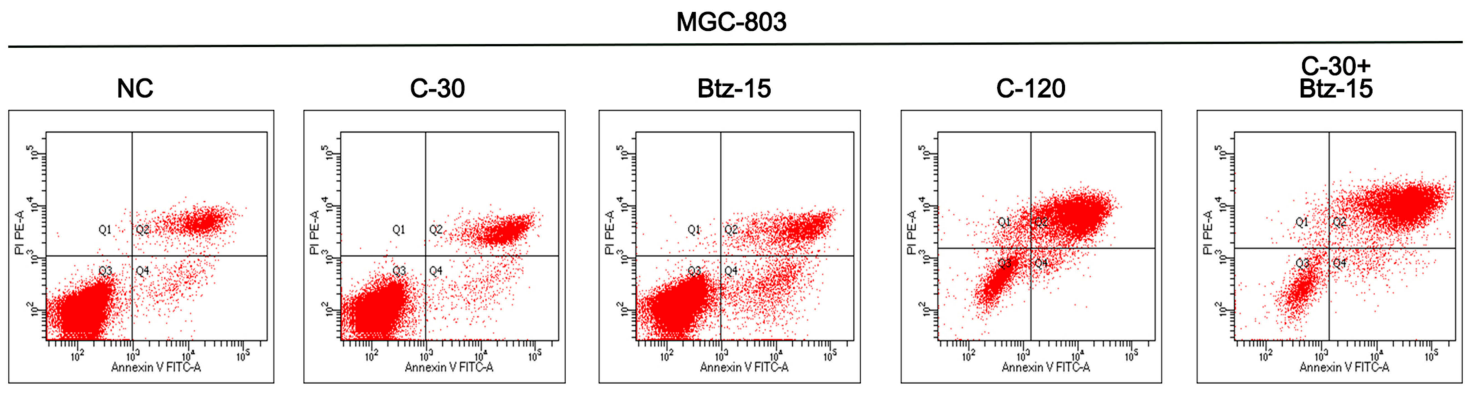

D
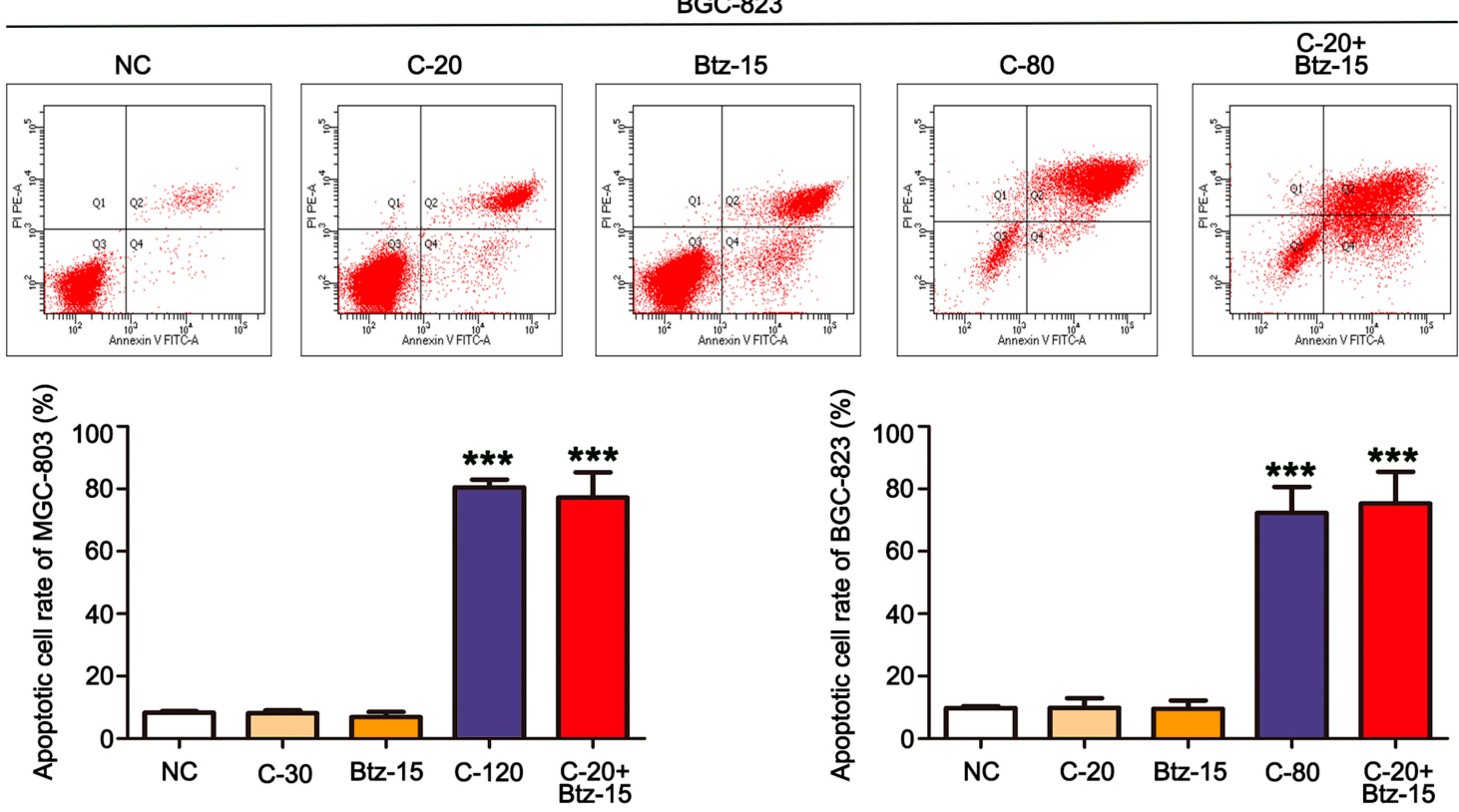

Figure 3 The combination of chidamide and bortezomib synergistically enhanced MGC-803 and BGC-823 cell apoptosis. The MGC-803 and BGC-823 cell lines were treated with chidamide ( $30 \mu \mathrm{M}$ for MGC-803 or $20 \mu \mathrm{M}$ for BGC-823), bortezomib (I5 nM), chidamide (I $20 \mu \mathrm{M}$ for MGC-803 or $80 \mu \mathrm{M}$ for BGC-823), or chidamide ( 30 $\mu \mathrm{M}$ for MGC-803 or $20 \mu \mathrm{M}$ for BGC-823) in combination with bortezomib (15 nM) for 48 hours. (A and B) Representative images of the expression of the apoptosisrelated proteins cleaved caspase-3, P53, and Bcl-2. (C and D) Flow cytometry of the number of cells in the apoptotic state after the different treatments, and their statistics. (One-way ANOVA with Bonferroni's post hoc test was applied to compare the indicated groups. $* * * P<0.00$ I, compared with the negative control, chidamide-, or bortezomib-alone groups). The experiment was repeated at least three times. Negative control: DMSO. 
A
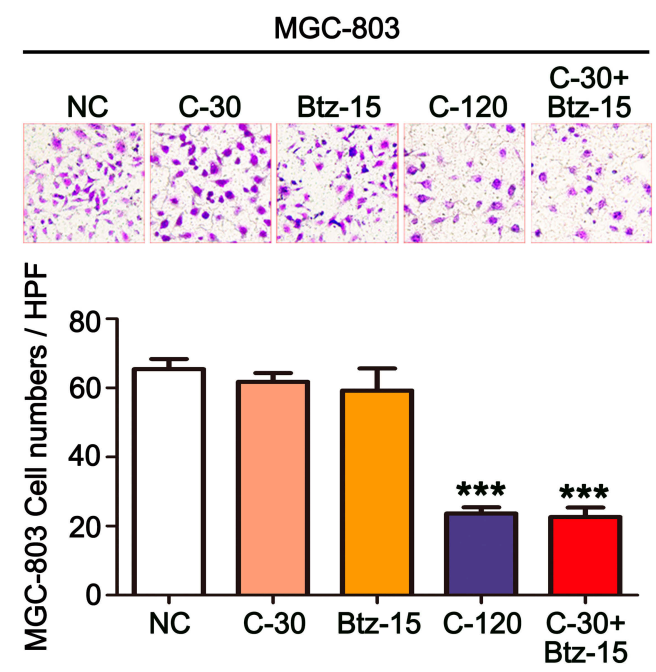

C
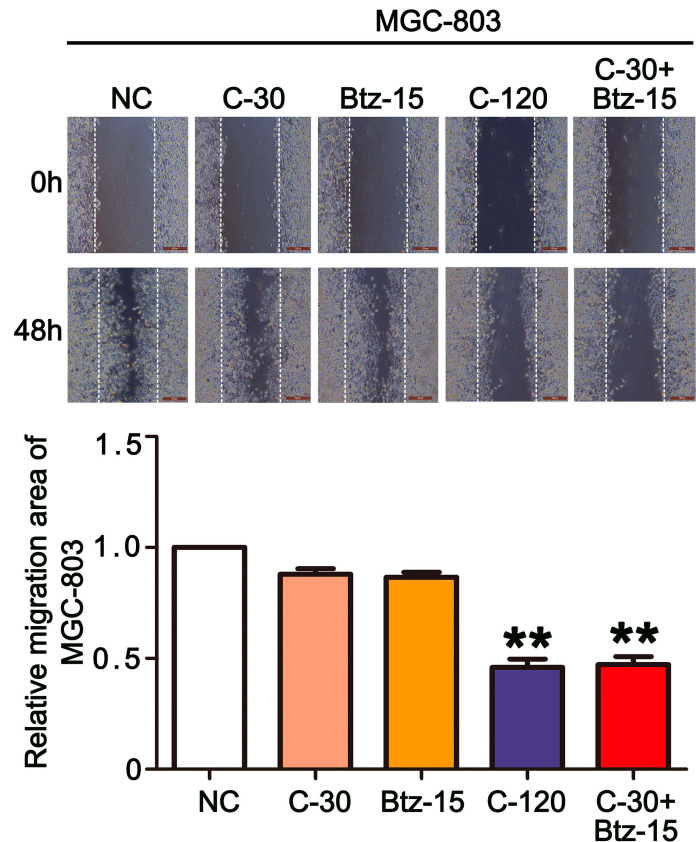

B
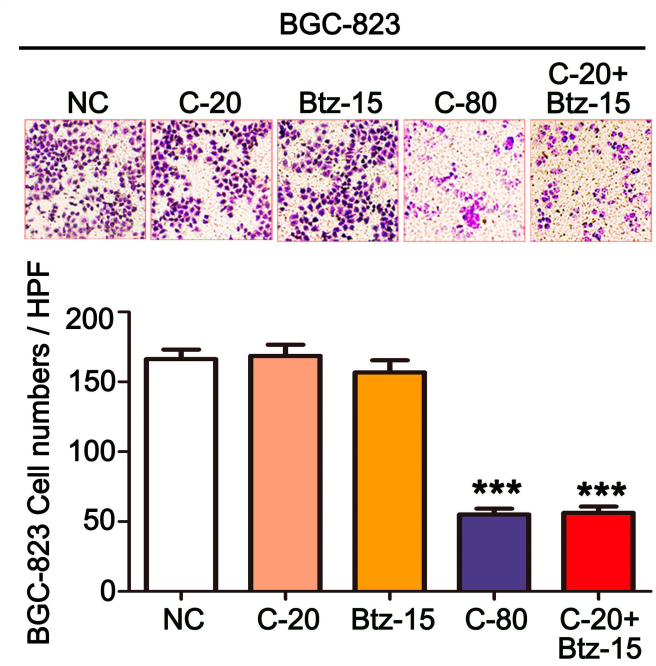

D
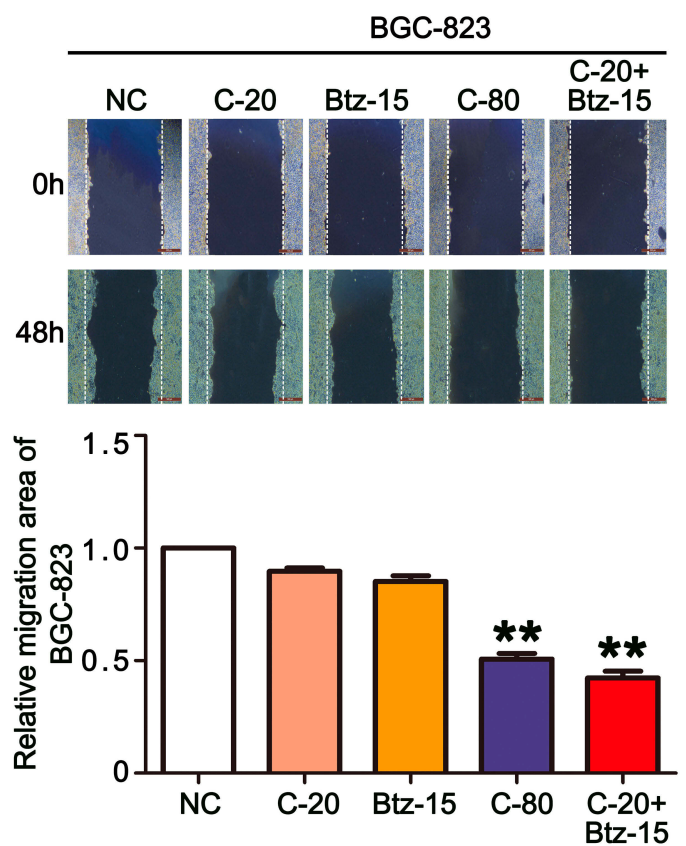

Figure 4 The combination of chidamide and bortezomib synergistically inhibited the migratory abilities of MGC-803 and BGC-823 cells. The MGC-803 and BGC-823 cell lines were treated with chidamide ( $30 \mu$ M for MGC-803 or $20 \mu$ for BGC-823), bortezomib (I5 nM), chidamide (I $20 \mu M$ for MGC-803 or $80 \mu M$ for BGC-823), or chidamide (30 $\mu$ M for MGC803 or $20 \mu \mathrm{M}$ for BGC-823) in combination with bortezomib (I5 nM) for 48 hours. (A and B) Representative images of the Transwell assay results at 24 hours after the different treatments (magnification $\times 100$ ). Migrated cells were quantified on the basis of the average of four randomly chosen high-power fields from the independent experiments performed in duplicate (One-way ANOVA with Bonferroni's posthoc test was applied to compare the indicated groups. $* * * P<0.00 \mathrm{I}$, compared with the negative control, chidamide-, or bortezomibalone groups). (C and D) Representative images and statistics of cell migration at 24 hours after scratching. Confluent MGC-803 and BGC-823 cell monolayers were wounded by scratching at 48 hours after the different treatments. Cell migration was monitored at 24 hours after wounding (the dotted line indicates the wound edge). The mean area migrated by the MGC-803 and BGC-823 cells was quantified (average of four independent microscope fields from three independent experiments each; One-way ANOVA with Bonferroni's posthoc test was applied to compare the indicated groups. ${ }^{*} * P<0.01$, compared with the negative control, chidamide-, or bortezomib-alone groups).

Low Dose of Bortezomib Enhances the Inhibition of HDAC Activity and PI3K/Akt Signaling Pathway by Chidamide

In order to further explore the detailed mechanism that bortezomib enhances the antitumor effect of chidamide. We first explored the effect of bortezomib on the inhibition of HDAC activity by chidamide. As a selective HDAC inhibitor, chidamide alone can significantly reduce MGC-803 and BGC-823 HDAC activity and the combined use of low-dose bortezomib can further enhance its inhibition of HDAC activity (Figure 6A and B). We further examined the effect of bortezomib on HDAC-mediated histone acetylation. As shown in Figure 6B 
A
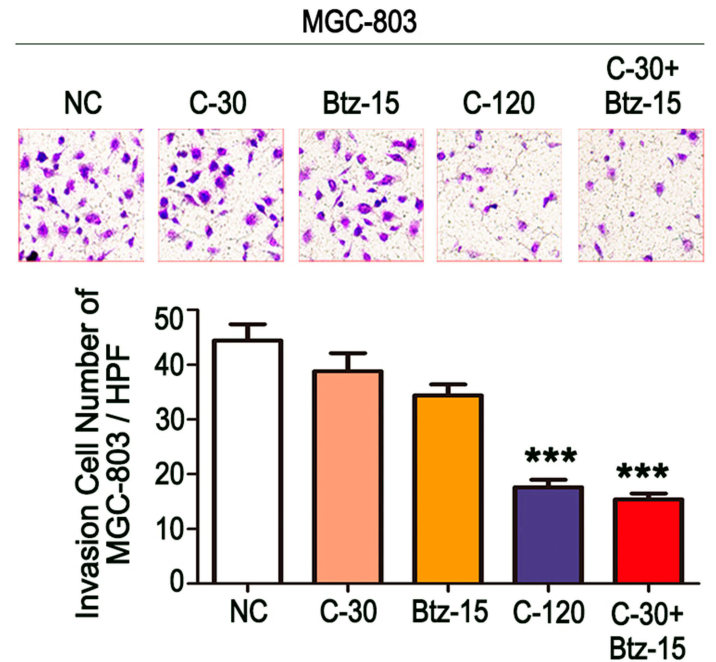

C

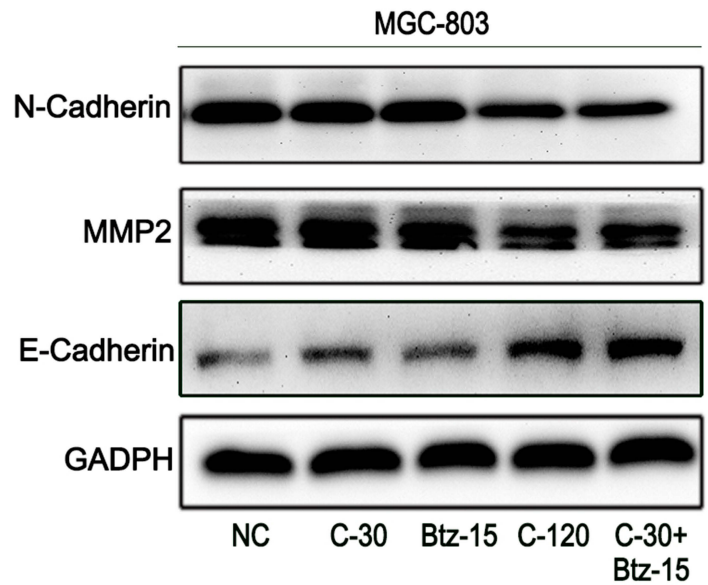

B
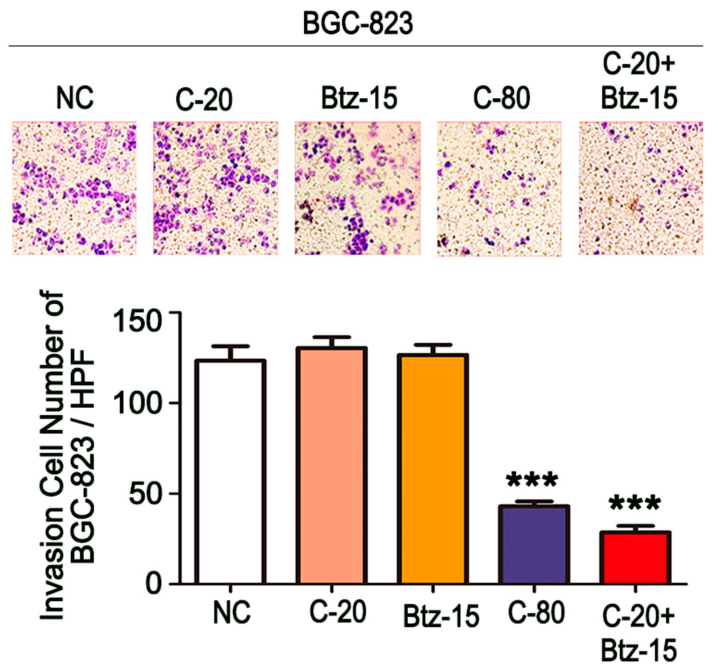

D

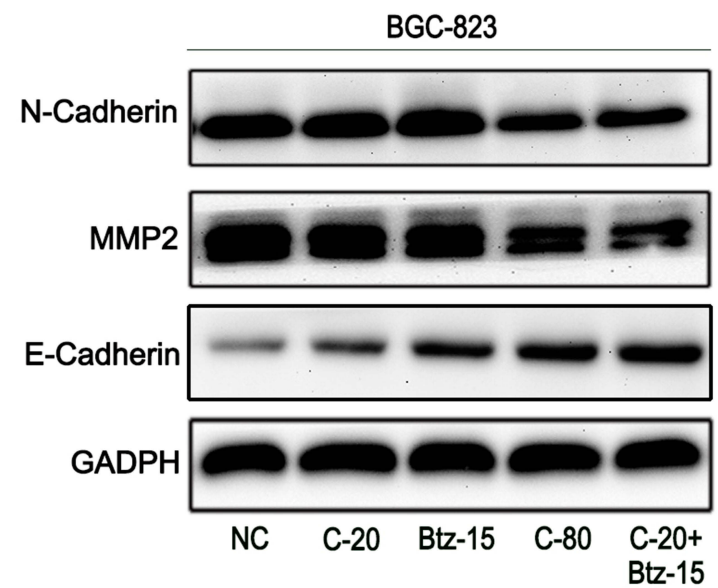

Figure 5 The combination of chidamide and bortezomib synergistically inhibited the invasive abilities of MGC-803 and BGC-823 cells. The MGC-803 and BGC-823 cell lines

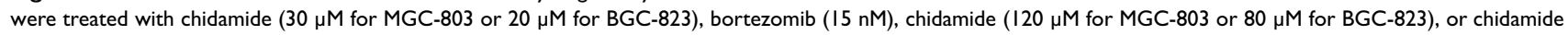
$(30 \mu \mathrm{M}$ for MGC-803 or $20 \mu \mathrm{M}$ for BGC-823) in combination with bortezomib ( $15 \mathrm{nM})$ for 48 hours. (A and B) Representative images of the cell invasion assay results at 24 hours after the different treatments (magnification $\times 100$ ). Invasive cells were quantified on the basis of the average of four randomly chosen high-power fields from the independent experiments performed in duplicate (One-way ANOVA with Bonferroni's posthoc test was applied to compare the indicated groups. $* * * P<0.00 \mathrm{I}$, compared with the negative control, chidamide-, or bortezomib-alone groups). (C and $\mathbf{D})$ Representative images of the expression of the invasion-related proteins $\mathrm{N}$-cadherin, MMP2, and VEGF.

and $\mathrm{C}$, the low-dose of bortezomib can significantly enhance the promotion of $\mathrm{H} 3$ and $\mathrm{H} 4$ acetylation by chidamide in the MGC-803 and BGC-823 cells. Previous studies have shown that chidamide can exert antitumor effects by activating the PI3K/Akt signaling pathway. ${ }^{24,25}$ Therefore, in order to clarify whether bortezomib regulates the antitumor effect of chidamide through PI3K/Akt, we examined the expression of total and phosphorylated PI3K and AKT. As shown in Figure 6A, low-dose bortezomib can significantly promote the inhibitory effect of chidamide on phosphorylated PI3K and phosphorylated AKT, but had no effect on the expression of total PI3K and $\mathrm{AKT}$ in the MGC-803 and BGC-823 cells (Figure 6E and F). These results suggested that bortezomib can enhance the inhibition of HDAC activity and PI3K/Akt signaling pathway by chidamide, thus enhancing its antitumor effect in the MGC-803 and BGC-823 cells.

\section{Antitumor Effects of Chidamide Alone or in Combination with Bortezomib on MGC-803 Cells in vivo}

Since chidamide combined with bortezomib showed stronger combined inhibition in the MGC-803 cell line in vitro, we 
A

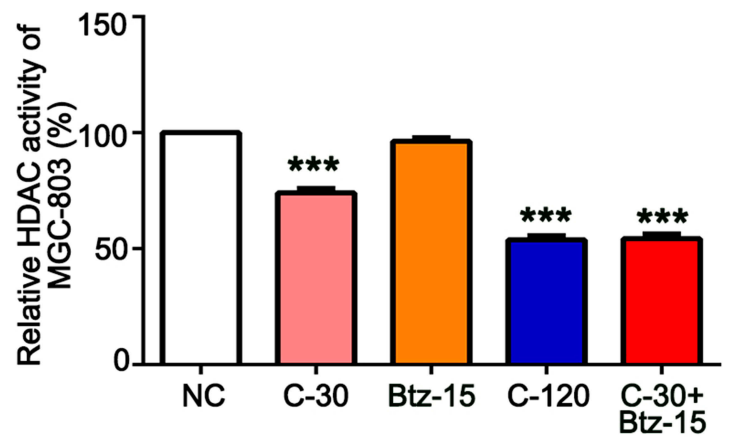

C

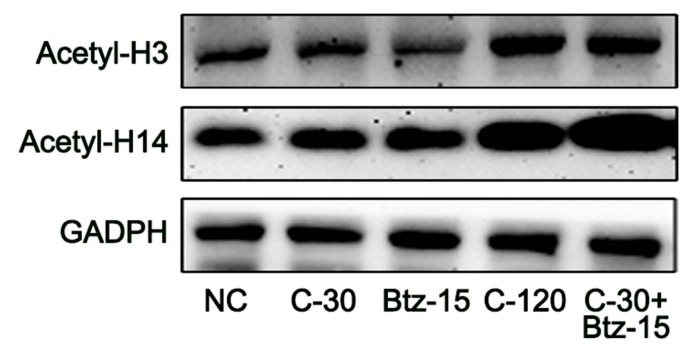

E

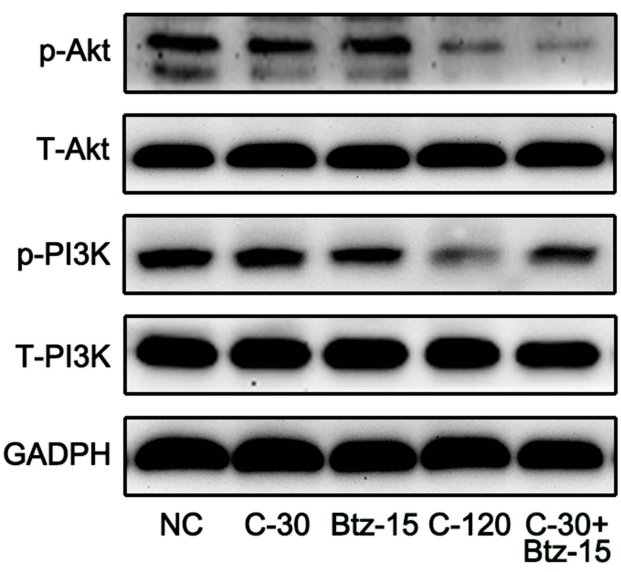

B

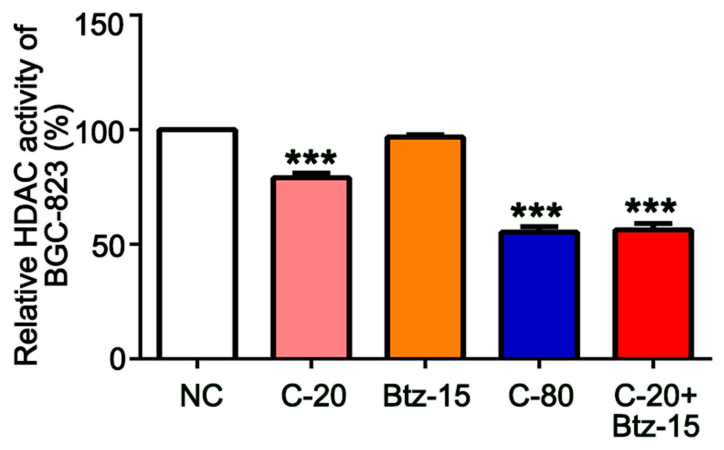

D

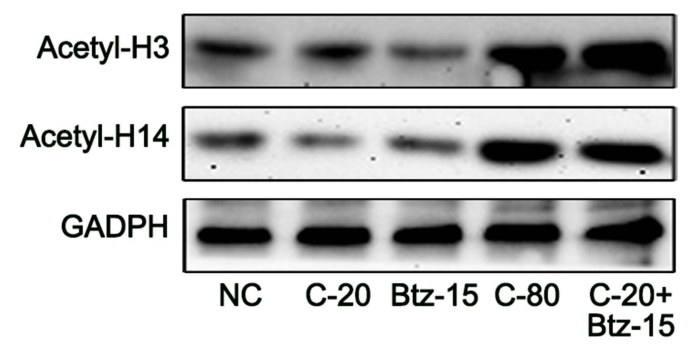

F

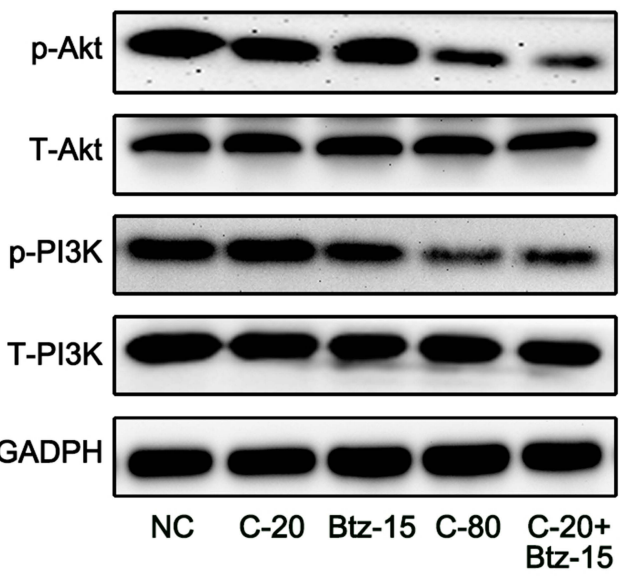

Figure 6 Low dose of bortezomib enhances the inhibition of HDAC activity and PI3K/Akt signaling pathway by chidamide. The MGC-803 and BGC-823 cell lines were treated with chidamide ( $30 \mu \mathrm{M}$ for MGC-803 or $20 \mu \mathrm{M}$ for BGC-823), bortezomib (I5 nM), chidamide (I20 $\mu$ M for MGC-803 or $80 \mu \mathrm{M}$ for BGC-823), or chidamide (30 $\mathrm{MM}$ for MGC-803 or $20 \mu$ M for BGC-823) in combination with bortezomib (I5 nM) for 48 hours. (A and B) The HDAC activities were tested and normalized to the NC group. (Experiments were performed in duplicate and One-way ANOVA with Bonferroni's posthoc test was applied to compare the indicated groups. $* * * P<0.00 \mathrm{I}$, compared with the negative control, chidamide-, or bortezomib-alone groups). (C and D) Representative images of the expression of the acetylated $\mathrm{H} 3$ and $\mathrm{H} 4$. (E and $\mathbf{F}$ ) Representative images of the expression of PI3K and AKT signal pathway-related proteins (total and phosphorylated PI3K and AKT).

chose MGC-803 for in vivo nude mice tumor formation experiments. The athymic nude mice bearing established MGC-803 tumor xenografts were treated via oral gavage with low-dose chidamide every day and bortezomib that was given intraperitoneally twice per week, either alone or in combination. The combination treatment significantly inhibited tumor growth as compared with the effects of PBS or the low doses of each drug alone $(P<0.05$, Figure 7A and B).
Moreover, the mice tolerated all treatments, indicating no significant weight loss or other apparent illness (Figure 7C). These findings were consistent with the in vitro results. Finally, in order to determine whether the combination of chidamide and bortezomib significantly increased toxicity, we performed HE staining of liver and kidney. We found that the combination of chidamide and bortezomib did not increase liver or kidney injury compared with giving each drug 
A

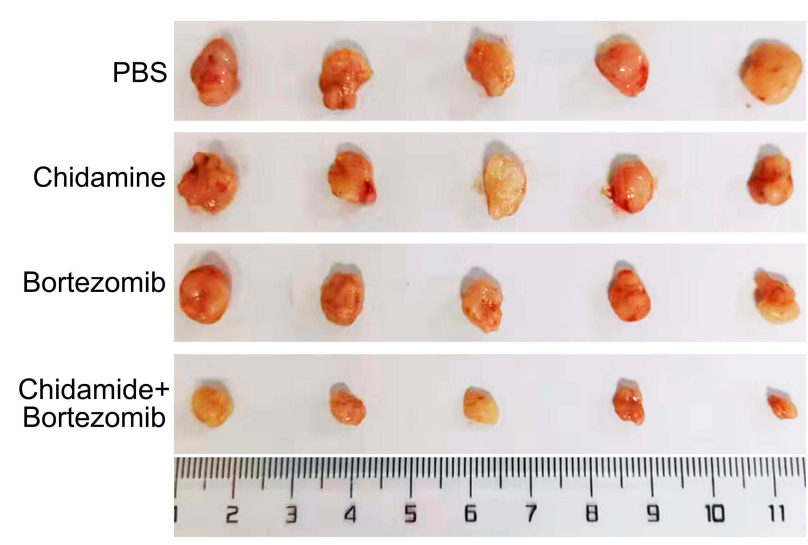

B

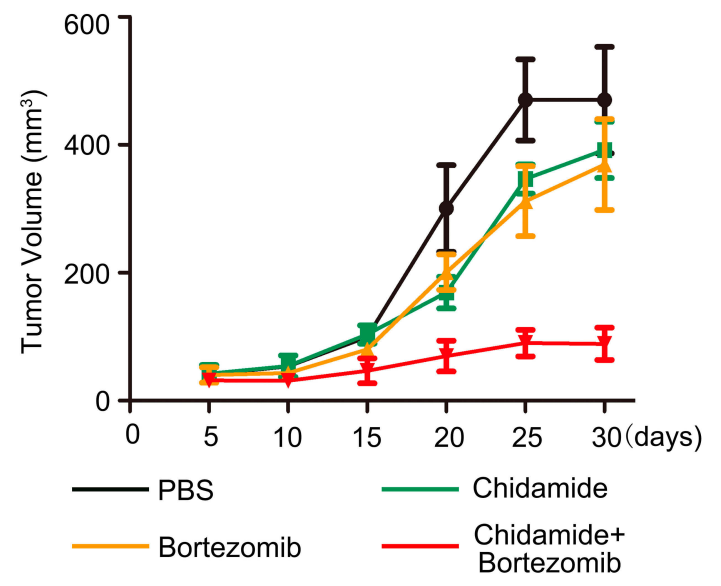

C

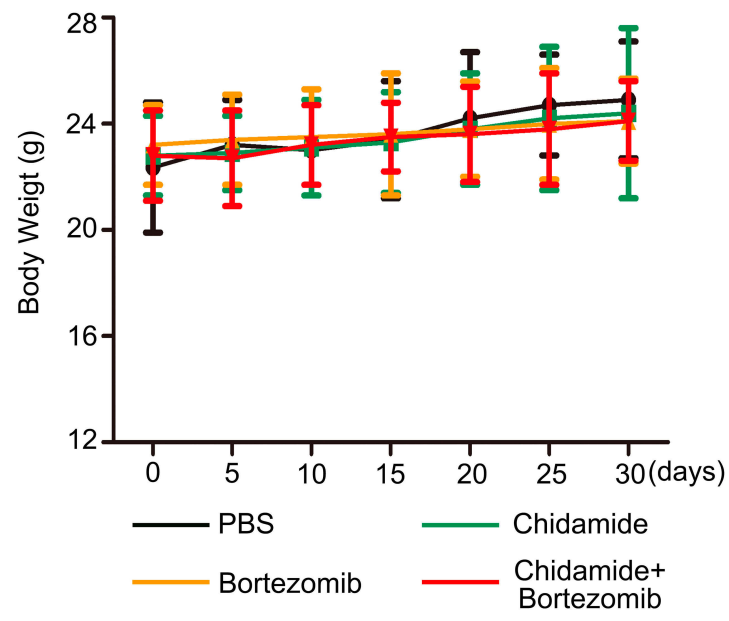

D

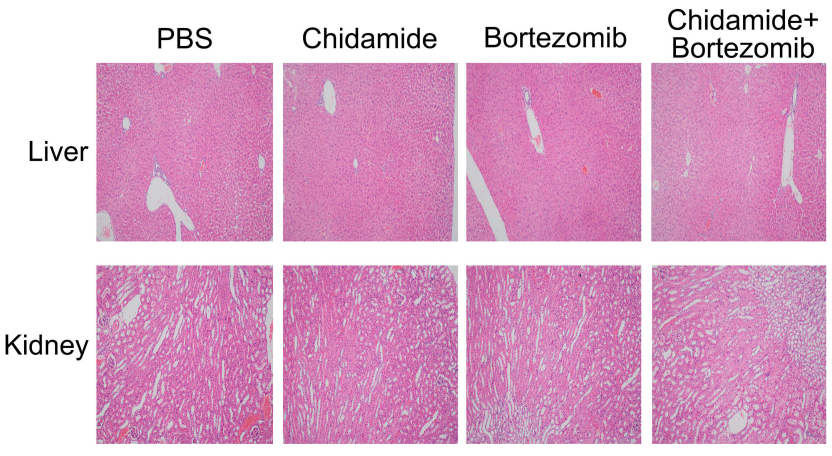

Figure 7 Antitumor effects of chidamide alone or in combination with bortezomib on MGC-803 cells in vivo. Nude mice bearing MGC-803 tumors ( $\square$ I00 mm ${ }^{3}$ ) were divided into four groups and treated with PBS, chidamide $\left(25 \mathrm{mg} \mathrm{kg}^{-1}\right.$ day $\left.{ }^{-1}\right)$, bortezomib $\left(0.5 \mathrm{mg} \mathrm{kg}{ }^{-1}\right.$, every 3 days $)$, or a combination of chidamide and bortezomib for 30 days. Tumor growth $(\mathbf{A})$, tumor volumes $(\mathbf{B})$ and body weight $(\mathbf{C})$ were measured. Results are shown as mean \pm SEM ( $n=5$ ). (D) Paraffin sections of kidney and liver of MGC-803 xenografts were stained with hematoxylin and eosin. Sections were examined by light microscopy (100 $\times$ magnification).

alone (Figure 7D), suggesting the safety of the combination of chidamide and bortezomib in vivo.

\section{Discussion}

In the current study, we demonstrated that a new generation of oral selective histone deacetylase inhibitor, chidamide, combined with proteasome inhibitor bortezomib, had synergistic effects on inhibiting proliferation, metastasis and promoting apoptosis of gastric cancer cell lines in vitro and in vivo. Mechanistically, the lowdose bortezomib cooperated with chidamide to inhibit the HDAC activity and PI3K/Akt signaling pathway, resulting in enhanced cellular apoptosis and anti-tumor effect. Since the low-dose of regent has low toxicity, combinated application of chidamide and bortezomib may provide a promising alternative to the treatment of gastric cancer.

In our study, we detected the viability of MGC-803 and BGC-823 cells treated with different treatment groups by CCK-8 assay, and revealed the significant differences between different treatments. Our findings are consistent with the previously published data of histone deacetylase inhibitors combined with bortezomib in other tumor cells, $^{26,27}$ suggesting that the combination of low-dose chidamide and bortezomib can significantly inhibit the activity of MGC-803 and BGC-823. This view has been further validated by EdU staining assay and nude mice tumorigenesis model. It provides a meaningful therapeutic strategy for reducing the dosage and adverse reactions of drugs in clinical. Besides (as shown in Figure 3C and D), 
the proportion of apoptotic cells labeled by connexin $\mathrm{V}$ is significantly higher than that of alone, which is more than additive, and these results are consistent with the phenomenon in leukemia. ${ }^{28}$ It has been reported that chidamide combined with other chemotherapeutics could play an anti-tumor role by regulating Bcl-2 and caspase-3 signaling pathways in the study of MDS, PTCL, and AML cell lines. $^{29,30}$ Furthermore, in vitro and in vivo studies have found that chidamide promotes mitochondrial apoptosis pathway-dependent apoptosis and inhibits the proliferation of pancreatic tumor cells by reducing the expression of bcl-2 and caspase- 3 and increasing the expression of bax and $\mathrm{p} 21 .^{31}$ According to this study, we found that both drugs induced the cleavage of caspase 3 and downregulated the expression of $\mathrm{Bcl}-2$ on $\mathrm{MGC}-803$ and BGC-823. Significantly, the combined effect of low-dose chidamide and bortezomib was better than that of alone, which mean that anticancer effect of low-dose chidamide and bortezomib, inducing apoptosis and mitigating migration and invasion, on MGC-803 and BGC-823 cells may partially via regulating caspase 3 and bcl-2 pathway. Some studies reported that Cyclin D1 is overexpressed in colorectal cancer and pancreatic cancer. ${ }^{32}$ Cyclin D1 activates CDK4 and CDK6, leading to the progression of G1-S in cancer cells. Moreover, cyclin E1 expression is also associated with increased malignant behavior in colorectal cancer, ovarian cancer, pancreatic cancer and various lymphomas. ${ }^{33-35}$ In the present study, cyclin D1 and cyclin E1 were significantly down-regulated after treatment with bortezomib and chidamide, which was consistent with the results of some current studies.

Distant metastasis is one of the main causes of tumorrelated death. ${ }^{33-35}$ Taylor et al found that the combination of histone deacetylase inhibitor SAHA and proteasome inhibitor bortezomib attenuated epithelial-mesenchymal conversion (EMT) inhibition of esophageal carcinoma by up-regulating E-cadherin and down-regulating the expression of N-cadherin. ${ }^{36-39}$ Similarly, we found that the combination of the low-dose chidamide and bortezomib significantly inhibited the ability of cell invasion and migration by regulating the EMT-related signal protein E-cadherin and N-cadherin, which was related to the down-regulation of the expression of metalloproteinase MMP2 that is associated with pro-oncogenic events, such as neo-angiogenesis, tumor cell proliferation, and metastasis. ${ }^{21}$ This is consistent with the results of our wound healing assay and Transwell migration assay.

The synergy between HDAC inhibitor and bortezomib has been well documented in previous studies, involving various mechanisms. Chadrick and colleagues demonstrated that bortezomib could enhance butyrate-induced NSCLC cell death by means of inhibiting $\mathrm{NF}-\kappa \mathrm{B}$ transcription. $^{23}$ In another study, combined treatment with bortezomib and MPT0G413 (a novel HDAC6selective inhibitor) leads to synergistic antiproliferative and proapoptotic effects in multiple myeloma cell lines through disturbing the bone marrow microenvironment and inducing caspase-dependent apoptosis. ${ }^{25}$ In our study, the low-dose of bortezomib can significantly enhance the promotion of $\mathrm{H} 3$ and $\mathrm{H} 4$ acetylation when combined with chidamide in the MGC-803 and BGC-823 cells, suggesting the enhanced HDAC-mediated histone acetylation in the combined therapy. More importantly, phosphorylated PI3K and phosphorylated AKT were found significantly decreased while the expression of total PI3K and AKT remained unchanged in combination with bortezomib and chidamide, indicating the involvement of PI3K/Akt signaling pathway in synergistic antitumor effect. To be noted, our study reveals the different mechanisms in the combined treatment of HDAC inhibitor and bortezomib. The inconsistent results may be explained by the use of different cell lines and proteasome inhibitors.

The hepatorenal toxicity of drugs has always been a problem that researchers pay close attention to when using chemotherapeutic drugs. Through the tumorforming model of nude mice in vivo, we confirmed that the tumor volume of the two drugs decreased significantly, but the body weight did not change significantly, and the liver and kidney HE staining confirmed that the combination of the two drugs did not produce the change of liver and kidney pathological changes, suggesting the safety and efficacy of this combination therapy. Accordingly, it is reasonable for us to assume that low-dose chidamide and bortezomib may be an alternative therapeutic approach for MGC-803 tumor treatment in vivo. However, this study was based on the use of a single proteasome inhibitor and HDAC inhibitor, other factors may lead to different results. We chose bortezomib and chidamide because they represent the most clinically significant drugs in their respective drug categories.

In summary, bortezomib combined with chidamide, rather than alone drug therapy, could effectively arrest the proliferation and metastasis of tumor cells and promote apoptosis. Thus, this combination therapy has clinical significance in providing a new perspective for the clinical formulation of drugs against gastric and esophageal cancers and warrants further research. 


\section{Data Sharing Statement}

The datasets used and/or analyzed during the current study are available from the corresponding author (sunkai@cellscience.org, Kai Sun) on reasonable request.

\section{Ethics and Consent Statement}

All experimental procedures were approved by the Animal Care Ethics Committee of the Zhengzhou University and Henan Provincial People's Hospital, and were performed in accordance with the American Physiology Society's "Guides for the Care and Use of Laboratory Animals" published by the National Institutes of Health.

\section{Funding}

This study was supported by the National Natural Science Foundation of China (No. 81971508, 81471589, 81273259); the Health Bureau of Henan Province (No. 201201005) and the Foundation and Frontier Research Grant of Henan Provincial Science and Technology Bureau (No. 142300410078 and No. 162102310293).

\section{Disclosure}

The authors report no conflicts of interest in this work.

\section{References}

1. Bray F, Ferlay J, Soerjomataram I, et al. Global cancer statistics 2018: GLOBOCAN estimates of incidence and mortality worldwide for 36 cancers in 185 countries. CA Cancer J Clin. 2018;68(6):394-424. doi:10.3322/caac. 21492

2. Petrillo A, Pompella L, Tirino G, et al. Perioperative treatment in resectable gastric cancer: current perspectives and future directions. Cancers. 2019;11(3):399. doi:10.3390/cancers11030399

3. Dillekas H, Rogers MS, Straume O. Are $90 \%$ of deaths from cancer caused by metastases? Cancer Med. 2019;8(12):5574-5576. doi:10.1002/ cam4.2474

4. Marks PA, Richon VM, Rifkind RA. Histone deacetylase inhibitors: inducers of differentiation or apoptosis of transformed cells. $J$ Natl Cancer Inst. 2000;92(15):1210-1216. doi:10.1093/jnci/92.15.1210

5. Mithraprabhu S, Kalff A, Chow A, et al. Dysregulated Class I histone deacetylases are indicators of poor prognosis in multiple myeloma. Epigenetics. 2014;9(11):1511-1520. doi:10.4161/15592294.2014. 983367

6. Mann BS, Johnson JR, Cohen MH, et al. FDA approval summary: vorinostat for treatment of advanced primary cutaneous T-cell lymphoma. Oncologist. 2007;12(10):1247-1252. doi:10.1634/theoncologist.12-101247

7. Shah RR. Safety and tolerability of histone deacetylase (HDAC) inhibitors in oncology. Drug Safety. 2019;42(2):235-245. doi:10.10 07/s40264-018-0773-9

8. San-Miguel JF, Hungria VT, Yoon SS, et al. Panobinostat plus bortezomib and dexamethasone versus placebo plus bortezomib and dexamethasone in patients with relapsed or relapsed and refractory multiple myeloma: a multicentre, randomised, double-blind phase 3 trial. Lancet Oncol. 2014;15(11):1195-1206. doi:10.1016/S1470-2045(14)70440-1
9. Liu L, Chen B, Qin S, et al. A novel histone deacetylase inhibitor Chidamide induces apoptosis of human colon cancer cells. Biochem Biophys Res Commun. 2010;392(2):190-195. doi:10.10 16/j.bbrc.2010.01.011

10. Wang H, Guo Y, Fu M, et al. Antitumor activity of Chidamide in hepatocellular carcinoma cell lines. Mol Med Rep. 2012;5 (6):1503-1508. doi:10.3892/mmr.2012.858

11. Zhou Y, Pan DS, Shan S, et al. Non-toxic dose chidamide synergistically enhances platinum-induced DNA damage responses and apoptosis in Non-Small-Cell lung cancer cells. Biomed Pharmacother. 2014;68(4):483-491. doi:10.1016/j.biopha.2014.03.011

12. Qiao Z, Ren S, Li W, et al. Chidamide, a novel histone deacetylase inhibitor, synergistically enhances gemcitabine cytotoxicity in pancreatic cancer cells. Biochem Biophys Res Commun. 2013;434 (1):95-101. doi:10.1016/j.bbrc.2013.03.059

13. Mao J, Li S, Zhao H, et al. Effects of chidamide and its combination with decitabine on proliferation and apoptosis of leukemia cell lines. Am J Transl Res. 2018;10(8):2567-2578.

14. Glickman MH, Ciechanover A. The ubiquitin-proteasome proteolytic pathway: destruction for the sake of construction. Physiol Rev. 2002;82(2):373-428. doi:10.1152/physrev.00027.2001

15. Almond JB, Cohen GM. The proteasome: a novel target for cancer chemotherapy. Leukemia. 2002;16(4):433-443. doi:10.1038/sj.leu. 2402417

16. Lenz HJ. Clinical update: proteasome inhibitors in solid tumors. Cancer Treat Rev. 2003;29 Suppl 1:41-48. doi:10.1016/S03057372(03)00082-3

17. Tew KD. Commentary on "Proteasome inhibitors: a novel class of potent and effective antitumor agents". Cancer Res. 2016;76 (17):4916-4917. doi:10.1158/0008-5472.CAN-16-1974

18. Kane RC, Farrell AT, SridharA R, et al. United States Food and Drug Administration approval summary: bortezomib for the treatment of progressive multiple myeloma after one prior therapy. Clinical Cancer Res. 2006;12(10):2955-2960. doi:10.1158/1078-0432.CCR06-0170

19. Fujita T, Doihara H, Washio K, et al. Antitumor effects and drug interactions of the proteasome inhibitor bortezomib (PS341) in gastric cancer cells. Anticancer Drugs. 2007;18(6):677-686. doi:10.1097/CAD.0b013e32808bf9d8

20. Saulle E, Petronelli A, Pasquini L, et al. Proteasome inhibitors sensitize ovarian cancer cells to TRAIL induced apoptosis. Apoptosis. 2007;12(4):635-655. doi:10.1007/s10495-006-0025-9

21. Liu X, Yue P, Chen S, et al. The proteasome inhibitor PS-341 (bortezomib) up-regulates DR5 expression leading to induction of apoptosis and enhancement of TRAIL-induced apoptosis despite up-regulation of c-FLIP and survivin expression in human NSCLC cells. Cancer Res. 2007;67(10):4981-4988. doi:10.1158/0008-5472. CAN-06-4274

22. Huang FI, Wu YW, Sung TY, et al. MPT0G413, A Novel HDAC6-selective inhibitor, and bortezomib synergistically exert anti-tumor activity in multiple myeloma cells. Front Oncol. 2019;9;249.

23. Denlinger CE, Keller MD, Mayo MW, et al. Combined proteasome and histone deacetylase inhibition in non-small cell lung cancer. J Thorac Cardiovasc Surg. 2004;127(4):1078-1086. doi:10.1016/ S0022-5223(03)01321-7

24. Zhang N, Liang C, Song W, et al. Antitumor activity of histone deacetylase inhibitor chidamide alone or in combination with epidermal growth factor receptor tyrosine kinase inhibitor icotinib in NSCLC. J Cancer. 2019;10(5):1275-1287. doi:10.7150/jca.28570

25. Xu F, Guo H, Shi M, et al. A combination of low-dose decitabine and chidamide resulted in synergistic effects on the proliferation and apoptosis of human myeloid leukemia cell lines. Am J Transl Res. 2019;11(12):7644-7655. 
26. Bhalla S, Balasubramanian S, David K, et al. PCI-24781 induces caspase and reactive oxygen species-dependent apoptosis through NF-kappa B mechanisms and is synergistic with bortezomib in lymphoma cells. Clinical Cancer Res. 2009;15(10):3354-3365. doi:10. 1158/1078-0432.CCR-08-2365

27. Adachi M, Zhang Y, Zhao X, et al. Synergistic effect of histone deacetylase inhibitors FK228 and m-carboxycinnamic acid bis-hydroxamide with proteasome inhibitors PSI and PS-341 against gastrointestinal adenocarcinoma cells. Clinical Cancer Res. 2004;10 (11):3853-3862. doi:10.1158/1078-0432.CCR-03-0806

28. Li Y, Chen K, Zhou Y, et al. A new strategy to target acute myeloid leukemia stem and progenitor cells using chidamide, a histone deacetylase inhibitor. Curr Cancer Drug Targets. 2015;15(6):493-503. doi:10.2174/156800961506150805153230

29. Zhao S, Guo J, Zhao Y, et al. Chidamide, a novel histone deacetylase inhibitor, inhibits the viability of MDS and AML cells by suppressing JAK2/STAT3 signaling. Am J Transl Res. 2016;8(7):3169-3178.

30. Shi Y, Dong M, Hong X, et al. Results from a multicenter, open-label, pivotal Phase II study of chidamide in relapsed or refractory peripheral T-cell lymphoma. Ann Oncol. 2015;26(8):1766-1771. doi:10. 1093/annonc/mdv237

31. Zhao B, HE T. Chidamide, a histone deacetylase inhibitor, functions as a tumor inhibitor by modulating the ratio of Bax/Bcl-2 and P21 in pancreatic cancer. Oncol Rep. 2015;33(1):304-310. doi:10.3892/ or. 2014.3595

32. Tay KC, Tan LT, Chan CK, et al. Formononetin: a review of its anticancer potentials and mechanisms. Front Pharmacol. 2019;10 (820). doi:10.3389/fphar.2019.00820.
33. Haddad RI, Weinstein LJ, Wieczorek TJ, et al. A phase II clinical and pharmacodynamic study of E7070 in patients with metastatic, recurrent, or refractory squamous cell carcinoma of the head and neck: modulation of retinoblastoma protein phosphorylation by a novel chloroindolyl sulfonamide cell cycle inhibitor. Clinical Cancer Res. 2004;10(14):4680-4687. doi:10.1158/1078-0432.CCR-04-0229

34. Roskoski R Jr. Cyclin-dependent protein serine/threonine kinase inhibitors as anticancer drugs. Pharmacol Res. 2019;139:471-488. doi:10.1016/j.phrs.2018.11.035

35. Harashima H, Dissmeyer N, SchnittGER A. Cell cycle control across the eukaryotic kingdom. Trends Cell Biol. 2013;23(7):345-356. doi:10.1016/j.tcb.2013.03.002

36. Polyak K, Weinberg RA. Transitions between epithelial and mesenchymal states: acquisition of malignant and stem cell traits. Nat Rev Cancer. 2009;9(4):265-273. doi:10.1038/nrc2620

37. Herzig M, Savarese F, Novatchkova M, et al. Tumor progression induced by the loss of E-cadherin independent of beta-catenin/Tcfmediated Wnt signaling. Oncogene. 2007;26(16):2290-2298. doi:10. 1038/sj.onc. 1210029

38. Taylor MD, Liu Y, Nagji AS, et al. Combined proteasome and histone deacetylase inhibition attenuates epithelial-mesenchymal transition through E-cadherin in esophageal cancer cells. J Thorac Cardiovasc Surg. 2010;139(5):1224-32, 32 e1. doi:10.1016/j.jtcvs.2010.01.039

39. Xing X, Tang YB, Yuan G, et al. The prognostic value of E-cadherin in gastric cancer: a meta-analysis [J]. Int $J$ Cancer. 2013;132 (11):2589-2596. doi:10.1002/ijc.27947

\section{Publish your work in this journal}

OncoTargets and Therapy is an international, peer-reviewed, open access journal focusing on the pathological basis of all cancers, potential targets for therapy and treatment protocols employed to improve the management of cancer patients. The journal also focuses on the impact of management programs and new therapeutic agents and protocols on patient perspectives such as quality of life, adherence and satisfaction. The manuscript management system is completely online and includes a very quick and fair peer-review system, which is all easy to use. Visit http://www.dovepress.com/ testimonials.php to read real quotes from published authors. 